\title{
The appropriate number of ELNs for lymph node negative breast cancer patients underwent MRM: a population-based study
}

\author{
Huiying Chi ${ }^{1}$, Chenyue Zhang ${ }^{2}$, Haiyong Wang ${ }^{3}$ and Zhehai Wang ${ }^{3}$ \\ ${ }^{1}$ Shanghai Geriatrics Institute of Traditional Chinese Medicine, Shanghai 200032, China \\ ${ }^{2}$ Department of Integrative Oncology, Fudan University Shanghai Cancer Center, Shanghai 200032, China \\ ${ }^{3}$ Department of internal Medicine-Oncology, Shandong Cancer Hospital and Institute, Shandong Cancer Hospital affiliated to \\ Shandong University, Shandong Academy of Medical Sciences, Jinan 250117, China
}

Correspondence to: Haiyong Wang, email: wanghaiyong6688@126.com

Zhehai Wang, email: wzhai8778@sina.com

Keywords: breast cancer, mastectomy, ELNs, lymph nodal negative, $X$-tile

Abbreviations: ELNs: examed lymph nodes, PLNs: positive lymph nodes, CSS: cancer specific survival, SEER: surveillance epidemiology and end results, NLNs: negative lymph nodes

Received: May 27, $2017 \quad$ Accepted: July 25, $2017 \quad$ Published: August 07, 2017

Copyright: Chi et al. This is an open-access article distributed under the terms of the Creative Commons Attribution License 3.0 (CC BY 3.0), which permits unrestricted use, distribution, and reproduction in any medium, provided the original author and source are credited.

\section{ABSTRACT}

Whether number of examed lymph nodes (ELNs) would bring survival benefit for patients with negative lymph nodes after modified radical mastectomy (MRM) is uncertain. In our study, using the Surveillance Epidemiology and End Results (SEER) database between 2004 and 2009, we screened the appropriate patients with negative lymph nodes underwent MRM. The Cox proportional hazard analysis was used to determine the effect of number of ELNs on cancer specific survival (CSS). The results showed that the number of ELNs was not an independent prognostic factor on CSS $(P=0.940)$. Then the $X$-tile mode was used to determine the appropriate threshold for ELNs count. The results showed that 9 was the appropriate cut-off point. Next, the logrank $x^{2}$ test was used to analyze the CSS based on different subgroup variables. The results showed that some subgroup variables including age $<50 / \geq 50$, grade I/III, AJCC T1/T2, ER positive/negative and PR positive/negative, demonstrated significant CSS benefits among the patients with the number of ELNs $\leq 9$ (all, $P<0.05$ ). However, three subgroup variables including grade II, AJCC T3 and AJCC T4, the patients with the number of ELNs $\leq 9$ did not bring significant CSS benefits (all, $P>0.1$ ). In conclusion, our study demonstrated that the number of ELNs was not an independent prognostic factor on CSS, and 9 can be selected as the appropriate cut-off point of ELNs for patients with negative lymph nodes who underwent MRM.

\section{INTRODUCTION}

Breast cancer is the most frequently diagnosed cancer and the leading cause of cancer death among females worldwide, with an estimated 1.7 million incidence and 521,900 mortalities in 2012 [1]. Breast cancer alone accounts for $25 \%$ of all cancers and $15 \%$ of all cancer-related deaths among females [1].

Axillary lymph node status has been widely acknowledged as an important prognostic factor for breast cancer patients underwent MRM [2-4]. It is well known that the number of positive lymph nodes (PLNs) is strongly associated with the tumor node metastasis (TNM) staging system and would determine the strategies of adjuvant therapy [5-8]. National Comprehensive Cancer Network (NCCN) guidelines and many studies have recommended post-mastectomy radiation therapy as a candidate therapy for breast cancer patients based on the number of PLNs [9-11]. Interestingly, other expression mode of lymph node status including the ratio of positive node defined as the number of PLNs divided by the total number of ELNs, the number of negative lymph nodes (NLNs) defined as the total number of ELNs by axillary dissection minus the number of PLNs, have also been introduced and even showed more significance in predicting the prognosis of breast cancer patients [12-15]. Importantly, the expression mode of lymph node is strongly dependent on the total number of ELNs. Theoretically, more number 
of ELNs may provide more accurate information on TNM classification and breast cancer prognosis by increasing the probability of PLNs. However, for the breast cancer patients with negative lymph nodes after MRM, whether more number of ELNs would bring survival benefit is uncertain. In addition, the appropriate number of ELNs should also be determined for these breast cancer patients.

To address these unknown issues of clinical significance, using the Surveillance, Epidemiology, and End Results (SEER)-registered database, we analyzed the association between the number of ELNs and prognosis of breast cancer patients. Importantly, we used an X-tile mode to determine an appropriate threshold for ELNs count.

\section{RESULTS}

\section{Patient demographics}

There were 15633 female breast cancer patients reported in the SEER database from 2004 to 2009. These patients were followed up for a consecutive 119 months. The clinical characteristics of all the patients were summarized in Table 1. The number of ELNs ranged from 1 to 81 . Most patients were white-raced (79.1\%) and diagnosed at the age of more than 50-year-old (74.1\%). the proportion of patients with Grade I, II and III was $19.8 \%, 41.0 \%$ and $39.2 \%$, respectively. Interestingly, most of the patients were diagnosed at the AJCC stage T1-2 $(91.3 \%)$. In addition, most patients were not received radiation $(87.7 \%)$, and the positive rates of ER and PR status were $72.1 \%$ and $61.9 \%$, respectively. The detailed characteristics were shown in Table 1.

\section{Number of ELNs and CSS}

After controlling for other prognostic factors including age, race, grade, AJCC $\mathrm{T}$ stage, radiation, ER status and PR status, the Cox proportional hazard analysis was used to analyze the correlation between the number of ELNs (as a continuous variable) and the CSS. Interestingly, our results showed that the number of ELNs was not an independent prognostic factor on CSS (hazard ratio (HR):1.000; confidence interval (CI) 95\%: 0.992 1.008; $P=0.940$ ) (Table 2). Next, we regarded the number of ELNs $=1$ as the reference, the Cox proportional hazard analysis was also used to analyze the correlation between the number of ELNs (as a categorical variable) and the CSS. Our results showed that the increased number of ELNs did not bring benefit for CSS (all, $P>0.1$ ) (Figure 1).

\section{Determine the appropriate cut-off point of ELNs count}

Since the number of ELNs was not an independent prognostic factor on CSS, it is necessary to identify the optimal cut-off count of lymph nodes for patients with negative lymph nodes. The X-tile mode was used to determine the appropriate cut-off point of ELNs count. Results showed 9 was the appropriate cut-off point (Figure 2A). The Kaplan-Meier analyses were used to generate the survival curves in X-tile mode, and the Log Rank test was applied to compare the prognosis between the patients with the number of ELNs $>9$ and the number of ELNs $\leq 9$. The results showed that the patients with the number of ELNs $\leq 9$ had a better CSS rate $(P<0.001)$ (Figure 2B and 2C).

\section{Validate the cut-off point of ELNs count}

In order to further verify the feasibility of the 9 as the appropriate cut-off point for ELNs count, we further analyzed individual number of ELNs from 1 to 15 using univariate log-rank test. The 5-year CSS with different numbers of ELNs were calculated, respectively. The results showed that the patients with more than nine ELNs count had a maximum log-rank $\chi^{2}$ value in CSS $\left(\chi^{2}=25.59, P<0.001\right)$ (Table 3$)$. Therefore, 9 can be selected as the appropriate cut-off point for these patients. The 5-year CSS rate of the patients with the number of more than nine ELNs count was 93.3\% (Table 3). The 5 -year CSS rate of the patients with the number of nine and fewer than nine ELNs count was 95.2\% (Table 3). Therefore, the cut-off 9 was validated as the appropriate threshold.

\section{Subgroup analysis for CSS based on the appropriate threshold}

The Cox proportional hazard analysis has shown that variables including age, grade, and AJCC T stage ER and PR status were all independent prognostic factors on CSS (Table 2). Next, subgroup analysis using the logrank $\chi^{2}$ test was conducted and the survival curves were formed based on the appropriate threshold. The results showed that the subgroup variables including age $<50$, age $\geq 50$, grade I, grade III, AJCC T1,AJCC T2, ER/PR positive and ER/PR negative, the number of ELNs $\leq 9$ all demonstrated significant CSS benefits (age $<50$ : logrank $\chi^{2}: 13.76, P<0.001$; age $\geq 50$ : log-rank $\chi^{2}: 13.24$, $P<0.001$; grade I: log-rank $\chi^{2}: 8.79, P=0.003$; grade III: log-rank $\chi^{2}: 8.02, P=0.005$; AJCC T1: log-rank $\chi^{2}: 8.90$, $P=0.003$; AJCC T2: log-rank $\chi^{2}: 4.51, P=0.034$; ER positive: log-rank $\chi^{2}: 26.78, P<0.001$; ER negative: logrank $\chi^{2}: 7.88, P=0.005$; PR positive: $\log$-rank $\chi^{2}: 12.00$, $P<0.001$; PR negative: log-rank $\chi^{2}: 7.19, P=0.007$ ) (Figure 3A-3J). However, three subgroup variables including grade II, AJCC T3 and AJCC T4, the number of ELNs $\leq 9$ didn't show significant CSS benefits (grade II: log-rank $\chi^{2}: 0.96 ; P=0.329$; AJCC T3: log-rank $\chi^{2}$ : $0.40 ; P=0.529$; AJCC T4: log-rank $\chi^{2}: 1.61 ; P=0.204$ ) (Supplementary Figure 1A-1C). 
Table 1: Characteristics of lymph node negative breast cancer patients underwent modified radical mastectomy from SEER Database from 2004-2009

\begin{tabular}{|c|c|}
\hline Variables & Number (\%) \\
\hline Total & $15633(100)$ \\
\hline Number of ELN & $1-81$ \\
\hline \multicolumn{2}{|l|}{ Age } \\
\hline$<50$ & $4042(25.9)$ \\
\hline$\geq 50$ & $11591(74.1)$ \\
\hline \multicolumn{2}{|l|}{ Race } \\
\hline White & $12365(79.1)$ \\
\hline Black & $1692(10.8)$ \\
\hline Others & $1576(10.1)$ \\
\hline \multicolumn{2}{|l|}{ Grade } \\
\hline I & $3103(19.8)$ \\
\hline II & $6403(41.0)$ \\
\hline III & $6127(39.2)$ \\
\hline \multicolumn{2}{|l|}{ T stage } \\
\hline $\mathrm{T} 1$ & $8769(56.1)$ \\
\hline $\mathrm{T} 2$ & $5496(35.2)$ \\
\hline $\mathrm{T} 3$ & $870(5.6)$ \\
\hline $\mathrm{T} 4$ & $498(3.2)$ \\
\hline \multicolumn{2}{|l|}{ Radiation } \\
\hline Yes & $1922(12.3)$ \\
\hline No & $13710(87.7)$ \\
\hline \multicolumn{2}{|l|}{ ER status } \\
\hline Positive & $11271(72.1)$ \\
\hline Negative & $4362(27.9)$ \\
\hline \multicolumn{2}{|l|}{ PR status } \\
\hline Positive & 9677 (61.9) \\
\hline Negative & $5956(38.1)$ \\
\hline
\end{tabular}

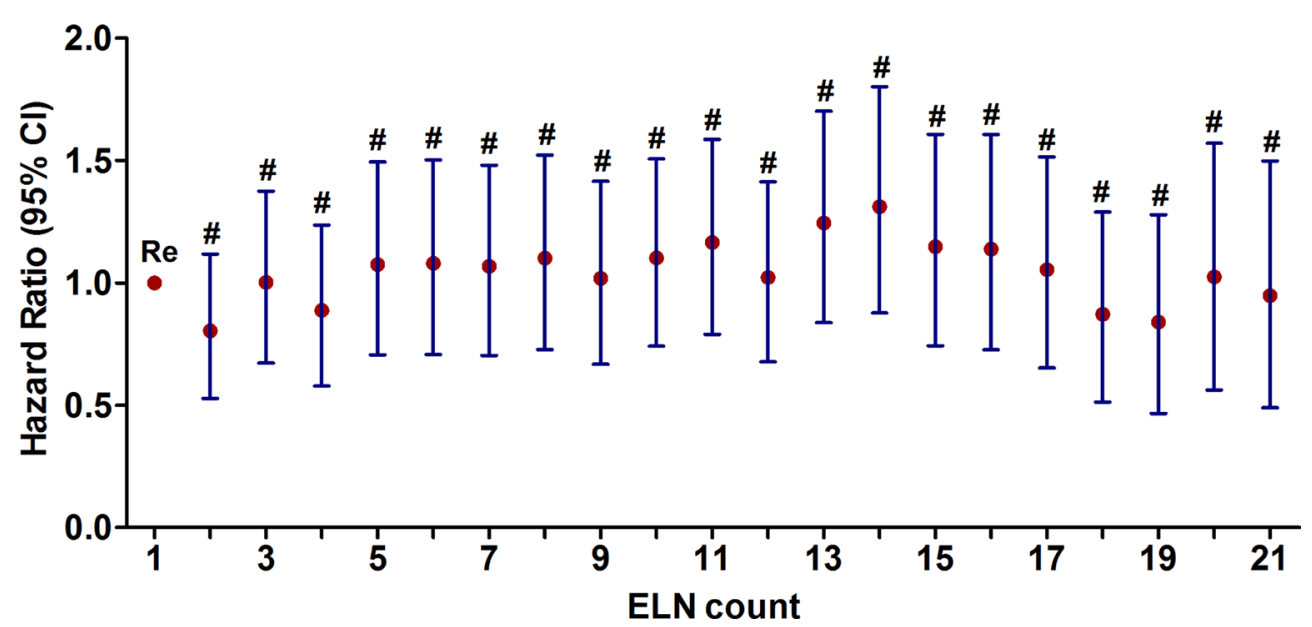

Figure 1: The correlation between the number of ELNs as a categorical variable and CSS adjusted for other variables using the Cox proportional hazard analysis. $\left({ }^{*} P>0.05\right)$. 
Table 2: Univariate and multivariate Cox regression analysis were used to evaluate the influence of different variables on CSS for lymph node negative breast cancer patients underwent modified radical mastectomy

\begin{tabular}{|c|c|c|c|c|}
\hline \multirow{2}{*}{ Variables } & \multicolumn{2}{|c|}{ Univariate analysis } & \multicolumn{2}{|c|}{ Multivariate analysis } \\
\hline & Wald $\chi^{2}$ & $P$ & HR (95\%CI) & $\boldsymbol{P}$ \\
\hline Number of ELN & 22.651 & $<0.001$ & $1.000(0.992-1.008)$ & 0.940 \\
\hline Age & 4.779 & 0.029 & & $<0.001$ \\
\hline$<50$ & & & reference & \\
\hline$\geq 50$ & & & $1.426(1.251-1.626)$ & $<0.001$ \\
\hline Race & 0.128 & 0.720 & Not included & \\
\hline \multicolumn{5}{|l|}{ White } \\
\hline \multicolumn{5}{|l|}{ Black } \\
\hline \multicolumn{5}{|l|}{ Others } \\
\hline Grade & 324.633 & $<0.001$ & & $<0.001$ \\
\hline I & & & reference & \\
\hline II & & & $2.026(1.551-2.647)$ & $<0.001$ \\
\hline III & & & $3.165(2.417-4.144)$ & $<0.001$ \\
\hline T stage & 707.957 & $<0.001$ & & $<0.001$ \\
\hline $\mathrm{T} 1$ & & & reference & \\
\hline $\mathrm{T} 2$ & & & $2.397(2.085-2.755)$ & $<0.001$ \\
\hline $\mathrm{T} 3$ & & & $3.825(3.097-4.725)$ & $<0.001$ \\
\hline $\mathrm{T} 4$ & & & $6.512(5.273-8.042)$ & $<0.001$ \\
\hline Radiation & 127.075 & $<0.001$ & & 0.998 \\
\hline Yes & & & reference & \\
\hline No & & & $1.000(0.856-1.169)$ & 0.998 \\
\hline ER status & 270.691 & $<0.001$ & & 0.020 \\
\hline Positive & & & reference & \\
\hline Negative & & & $1.224(1.033-1.452)$ & 0.020 \\
\hline PR status & 240.919 & $<0.001$ & & 0.001 \\
\hline Positive & & & reference & \\
\hline Negative & & & $1.323(1.119-1.565)$ & 0.001 \\
\hline
\end{tabular}

\section{DISCUSSION}

Theoretically, more number of ELNs may provide accurate information on TNM classification and prognosis in breast cancer patients by increasing the probability of PLNs. In fact, some studies have demonstrated that a strong correlation between the number of ELNs and survival benefit of patients as described $[16,17]$. However, these studies are mainly based on the patients with positive lymph nodes. For the breast cancer patients with negative lymph nodes after MRM, our results demonstrated that the increased number of ELNs was not an independent prognostic factor on CSS and did not bring CSS benefit, which were also supported by other studies [18-20]. For instance, moorman et al demonstrated that the number of ELNs was found to be associated with neither 5-year nor long-term survival; the proportion of women dying from breast cancer was the same in both groups (those patients with $>$ or $=20$ lymph nodes examined vs. those in whom $<$ 20 lymph nodes were examined) [18]. Interestingly, Camp et al. even find a worse 5-year survival for patients with tumor free lymph nodes more than 20 compared with patients with fewer than 20 tumor free lymph nodes [20]. However, some studies have inconsistent conclusion. For example, a study by van der wal et al showed that node-negative patients with $<14$ lymph nodes removed had a 10 year survival of $79 \%$ compared with $89 \%$ in patients with $>$ or $=$ 14 lymph nodes removed [21]. Another study by Krag et al showed that even when all regional lymph nodes are pathologically negative, the number of nodes removed 


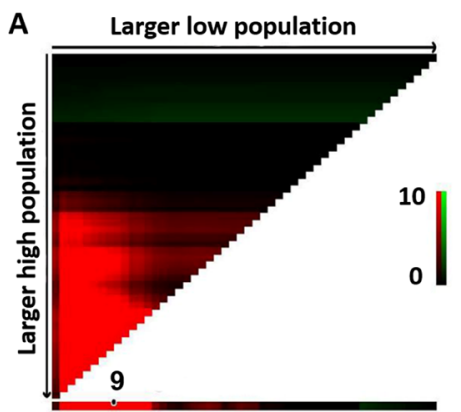

C

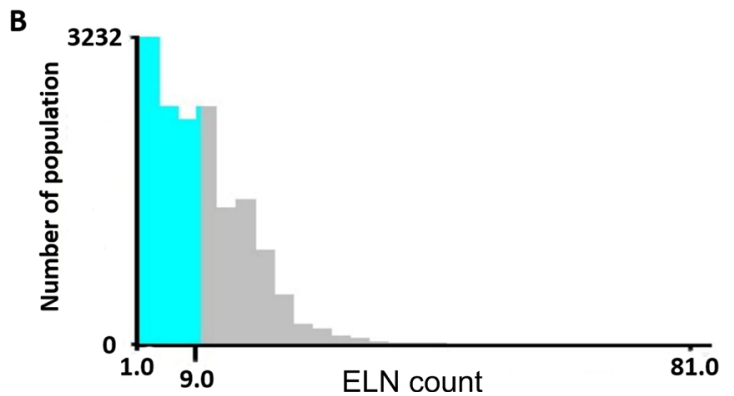

Figure 2: The appropriate cut-off point of ELNs count using the X-tile model. (A) X-tile plots of matched number of ELNs. (B) The optimal cut-off point highlighted by the gray and green panel. (C) The CSS curves between the patients with the number of ELNs $>9$ and $\leq 9(P<0.001)$.

A

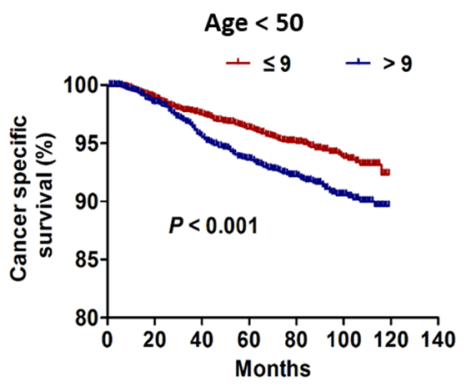

D

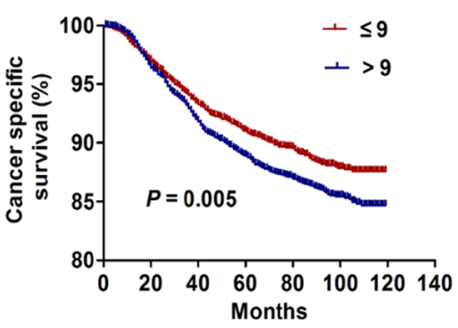

B

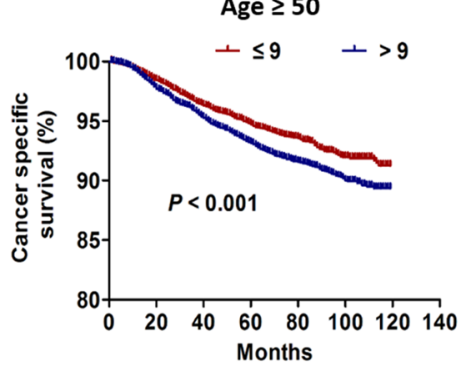

E

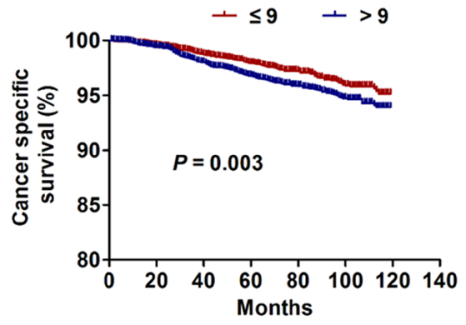

C

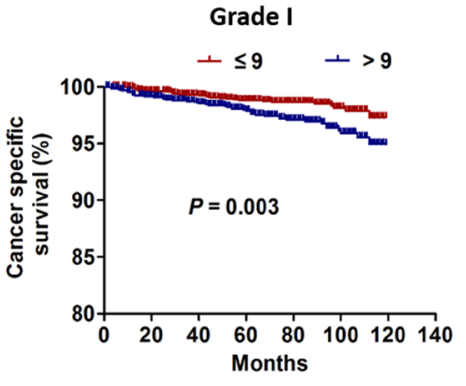

F

AJCC T2
G

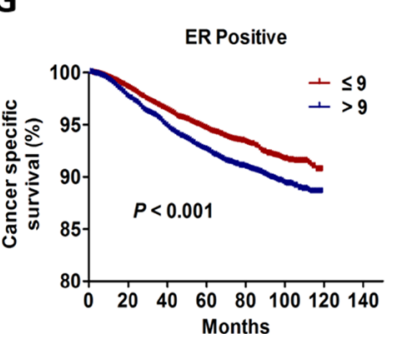

H

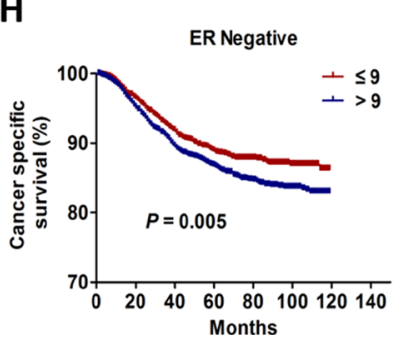

I

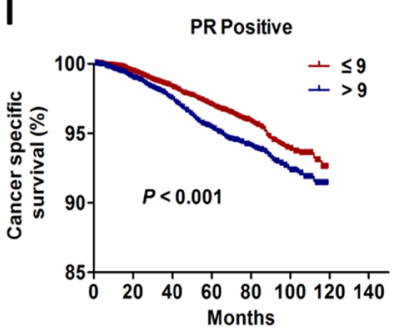

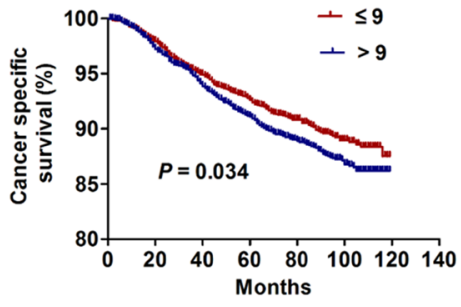

J

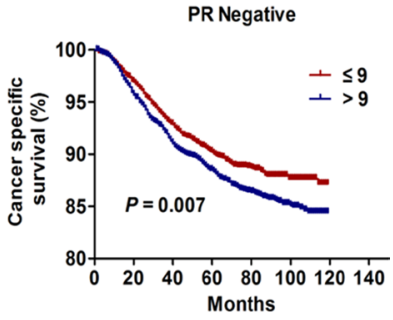

Figure 3: The CSS curves between the patients with the number of ELNs $>9$ and $\leq \mathbf{9}$ according to different variables. (A) The CSS curves of patients aged $<50(P<0.001)$. (B) The CSS curves of patients aged $\geq 50(P<0.001)$. (C) The CSS curves of patients with grade I $(P=0.003)$. (D) The CSS curves of patients with grade III $(P=0.005)$. (E) The CSS curves of patients with AJCC T1 $(P=0.003)$. (F) The CSS curves of patients with AJCC T2 $(P=0.034)$. (G) The CSS curves of patients with ER positive $(P<0.001)$. (H)The CSS curves of patients with ER negative $(P=0.005)$. (I) The CSS curves of patients with PR positive $(P<0.001)$. (J) The CSS curves of patients with PR negative $(P=0.007)$. 
Table 3: The log rank $\chi^{2}$ test to evaluate the influence of difference cut-off point on CSS for lymph node negative breast cancer patients underwent modified radical mastectomy

\begin{tabular}{|c|c|c|c|c|}
\hline Cutoff & Number & 5-year CSS (\%) & Log rank $\chi^{2}$ & $P$ \\
\hline$\leq 1$ & 943 & 94.8 & 2.25 & 0.134 \\
\hline$>1$ & 14690 & 94.2 & & \\
\hline$\leq 2$ & 2116 & 96.1 & 13.95 & $<0.001$ \\
\hline$>2$ & 13517 & 94.0 & & \\
\hline$\leq 3$ & 3232 & 95.8 & 17.90 & $<0.001$ \\
\hline$>3$ & 12401 & 93.8 & & \\
\hline$\leq 4$ & 4162 & 95.9 & 24.61 & $<0.001$ \\
\hline$>4$ & 11471 & 93.7 & & \\
\hline$\leq 5$ & 4944 & 95.7 & 23.56 & $<0.001$ \\
\hline$>5$ & 10689 & 93.6 & & \\
\hline$\leq 6$ & 5729 & 95.5 & 24.01 & $<0.001$ \\
\hline$>6$ & 9904 & 93.5 & & \\
\hline$\leq 7$ & 6531 & 95.4 & 23.86 & $<0.001$ \\
\hline$>7$ & 9102 & 93.4 & & \\
\hline$\leq 8$ & 7344 & 95.2 & 24.89 & $<0.001$ \\
\hline$>8$ & 8289 & 93.4 & & \\
\hline$\leq 9$ & 8093 & 95.2 & 25.59 & $<0.001$ \\
\hline$>9$ & 7540 & 93.3 & & \\
\hline$\leq 10$ & 8955 & 95.0 & 22.18 & $<0.001$ \\
\hline$>10$ & 6678 & 93.2 & & \\
\hline$\leq 11$ & 9771 & 94.9 & 14.23 & $<0.001$ \\
\hline$>11$ & 5862 & 93.1 & & \\
\hline$\leq 12$ & 10589 & 94.8 & 15.58 & $<0.001$ \\
\hline$>12$ & 5044 & 93.1 & & \\
\hline$\leq 13$ & 11348 & 94.7 & 9.96 & 0.002 \\
\hline$>13$ & 4285 & 93.0 & & \\
\hline$\leq 14$ & 12022 & 94.6 & 4.92 & 0.027 \\
\hline$>14$ & 3611 & 93.2 & & \\
\hline$\leq 15$ & 12602 & 94.5 & 3.00 & 0.083 \\
\hline$>15$ & 3031 & 93.3 & & \\
\hline
\end{tabular}

is associated with survival; In the group of negativenode breast cancer patients, the increased number of removed nodes is associated with improved survival; The hazard rate for death in the node-negative group was roughly $5 \%$ less for each additional five nodes removed [17]. The reason for the difference may be attributed to the fact that only patients undergoing MRM were enrolled in our study.

We have demonstrated that increasing number of ELNs did not bring any survival benefit. Therefore, it is important to determine the appropriate number of ELNs. We therefore used X-tile model to determine the optimal cutoff value. X-tile plots provide a single, global assessment of every possible way of dividing a population into low-, medium-, and high- level marker expression [22]. In addition, X-tile can produce corrected $p$ values using several Monte Carlo simulations: Cross-Validation takes our dataset, randomly splits it into two halves, finds the optimal cut-point of one half, and then divides the other half according to this cut-point. Then, it finds the optimal cut-point of the second half and similarly divides the first. [22]. The X-tile plots have been applied by other study to determine the appropriate cut-off value [23]. Our results demonstrated that 9 can be selected as the appropriate cutoff point for these patients. In addition, using the minimum $p$ value method used in others references [24, 25], we 
further verify the feasibility of 9 as appropriate cut-off point for ELNs count. In fact, a study from Danish Breast Cancer Cooperative Group in 1992 has demonstrated that the number of lymph nodes removed should be at least up to 10 to exclude misclassification of node-positive patients as node negative [16]. However, to the best of our knowledge, we first developed a cut-off for ELNs among a huge group of breast cancer patients. Our findings might give some advice to specialists in surgery to determine the extent of lymph node dissection.

Importantly, in our study, the log-rank $\chi^{2}$ test was applied and subgroup analysis was conducted. It is worth noting that for the subgroup patients with AJCC T3 and T4, the CSS difference was not found between the patients with $\leq 9$ ELNs and $>9$ ELNs. In fact, some studies have demonstrated that in breast cancer patients, there is a strong correlation between tumor size and the risk of axillary lymph nodes involvement [26-28]. For the subgroup patients with AJCC T3 and T4, it was not appropriate to regard nine as the cut-off point of ELNs. Therefore, further studies are needed to determine the number of ELNs in combination with other prognostic factors among breast cancer patients. The ELNs rate should be further assessed, especially for the high-risk patients.

Undeniably, this study also has several limitations.

Firstly, as the non-randomized study, the intrinsic defects exist in any retrospective study despite a relatively larger sample size. Secondly, some important prognosis factors such as HER-2, Ki67 status were not included in the present study.

In conclusion, our study demonstrated that the number of ELNs was not an independent prognostic factor on CSS. Importantly, our results demonstrated that 9 can be selected as the appropriate cut-off point of ELNs for patients with negative nodes who underwent modified radical mastectomy. Of course, further randomized controlled studies and sufficient subgroup analyses are warranted to validate our conclusion.

\section{MATERIALS AND METHODS}

\section{Patient selection}

The SEER Cancer Statistics Review (http://seer. cancer.gov/data/citation.html) is published annually by the Data Analysis and Interpretation Branch of the National Cancer Institute, MD, USA. A total of 18 population-based cancer registries in the United States were included in the current SEER database [29]. The SEER*Stat software (SEER*Stat 8.3.2) was used to identify the appropriate patients. Using this software, we screened female breast cancer patients whose histological type are limited to ductal and lobular neoplasms between January 1, 2004 and December 31, 2009. The included patients should meet the following criteria: the diagnosis was confirmed microscopically, they should be female with the confirmed age, active follow-up and only one primary tumor. In addition, the patients should be those who have received modified radical mastectomy, with no positive lymph nodes removed. Patients with benign or borderline tumors were excluded. And patients lacking information on age, race, grade, AJCC T stage, ER status and PR status, the number of examed lymph nodes, cause of death, survival months were also excluded.

\section{Ethics statement}

This study was mainly based on the SEER database and was conducted in compliance with the Helsinki Declaration. We obtained permission to access the files of SEER program research data and the reference number is 11304-Nov 2015. The informed consent was not required because personal identifying information was not involved. This study was approved by the ethics committee of the Shandong Cancer Hospital affiliated to Shandong University.

\section{Statistical analysis}

For all the patients, the following variables were analyzed: Age, race, grade, AJCC T stage, radiation, ER status and PR status, and the number of ELNs. In addition, CSS were regarded as the primary endpoint of this study and extracted from the SEER database. CSS is a survival measure representing survival of a specified cancer of death in the absence of other causes of death. The Cox proportional hazard analysis was used to determine the effect of number of ELNs on CSS adjusted for other significant prognostic factors. The number of ELNs is actually the number of lymph nodes removed after operation. The X-tile mode was used to determine the appropriate threshold for ELNs count. Then based on the appropriate threshold, the log-rank $\chi 2$ test was also performed to analyze the CSS based on different subgroup variables. In addition, the appropriate threshold was validated by using Log rank $\chi^{2}$ test based on different cut-off values. All statistical tests were two-sided, and a $P<0.05$ was considered statistically significant. The statistical software SPSS 18.0 (SPSS, Chicago, IL, USA) was used for all data analyses.

\section{Author contributions}

Huiying Chi conducted data collection and statistical analysis; Chenyue Zhang wrote the manuscript; Haiyong Wang and Zhehai Wang conducted the overall design and modification of manuscript.

\section{CONFLICTS OF INTEREST}

All authors have reviewed the final version of the manuscript and approve it for publication. The authors have no conflicts of interest to declare. 


\section{FUNDING}

This study was supported jointly by the National Natural Science Foundation of China (No. 81603348); China Postdoctoral fund (No. 21300075311104) and Shandong postdoctoral innovation special fund (No. 201602012).

\section{REFERENCES}

1. Torre LA, Bray F, Siegel RL, Ferlay J, Lortet-Tieulent J, Jemal A. Global cancer statistics, 2012. CA Cancer J Clin. 2015; 65:87-108.

2. Carter CL, Allen C, Henson DE. Relation of tumor size, lymph node status, and survival in 24,740 breast cancer cases. Cancer. 1989; 63:181-187.

3. Mwakigonja AR, Rabiel H, Mbembati NA, Lema LE. The pattern of prognostic and risk indicators among women with breast cancer undergoing modified radical mastectomy in Dar es Salaam, Tanzania. Infect Agent Cancer. 2016; 11:28.

4. Vinh-Hung V, Cserni G, Burzykowski T, van de Steene J, Voordeckers M, Storme G. Effect of the number of uninvolved nodes on survival in early breast cancer. Oncol Rep. 2003; 10:363-368.

5. Escobar PF, Patrick RJ, Rybicki LA, Weng DE, Crowe JP. The 2003 revised TNM staging system for breast cancer: results of stage re-classification on survival and future comparisons among stage groups, Ann Surg Oncol. 2007: 14:143-147.

6. Wu SG, Chen Y, Sun JY, Li FY, Lin Q, Lin HX, He ZY. Using the lymph nodal ratio to predict the risk of locoregional recurrence in lymph node-positive breast cancer patients treated with mastectomy without radiation therapy. Radiat Oncol. 2013; 8:119.

7. Wang H, Kong L, Zhang C, Chen D, Zhu H, Yu J. Should all breast cancer patients with four or more positive lymph nodes who underwent modified radical mastectomy be treated with postoperative radiotherapy? A populationbased study. Oncotarget. 2016; 7:75492-75502. http://doi. org/10.18632/oncotarget.12260.

8. Lin J, Li C, Zhang C, Shi F, Wang H. Postmastectomy radiation therapy for breast cancer patients with one to three positive lymph nodes: a propensity score matching analysis. Future Oncol. 2017; 13:1395-1404.

9. Jagsi R. Progress and controversies: radiation therapy for invasive breast cancer. CA Cancer J Clin. 2014; 64:135-152.

10. Stecklein SR, Shen X, Mitchell MP. PostMastectomy Radiation Therapy for Invasive Lobular Carcinoma: A Comparative Utilization and Outcomes Study. Clin Breast Cancer. 2016; 16:319-326.

11. Rusthoven CG, Rabinovitch RA, Jones BL, Koshy M, Amini A, Yeh N, Jackson MW, Fisher CM. The Impact of Postmastectomy and Regional Nodal Radiation after Neoadjuvant Chemotherapy for Clinically Lymph Node Positive Breast Cancer: A National Cancer Database (NCDB) Analysis. Ann Oncol. 2016; 27:818-827.
12. Chagpar AB, Camp RL, Rimm DL. Lymph node ratio should be considered for incorporation into staging for breast cancer. Ann Surg Oncol. 2011; 18:3143-3148.

13. Dings PJ, Elferink MA, Strobbe LJ, de Wilt JH. The prognostic value of lymph node ratio in node-positive breast cancer: a Dutch nationwide population-based study. Ann Surg Oncol. 2013; 20:2607-2614.

14. Duraker N, Batı B, Caynak ZC, Demir D. Lymph node ratio may be supplementary to TNM nodal classification in node-positive breast carcinoma based on the results of 2,151 patients. World J Surg. 2013; 37:1241-1248.

15. Wang H, Zhang C, Kong L, Zhu H, Yu J. Better survival in PMRT of female breast cancer patients with $>5$ negative lymph nodes: A population-based study. Medicine (Baltimore). 2017; 96:e5998.

16. Axelsson CK, Mouridsen HT, Zedeler K. Axillary dissection of level I and II lymph nodes is important in breast cancer classification. The Danish Breast Cancer Cooperative Group (DBCG). Eur J Cancer. 1992; 28A:1415-1418.

17. Krag DN, Single RM. Breast cancer survival according to number of nodes removed. Ann Surg Oncol. 2003; 10:1152-1159.

18. Moorman PG, Hamza A, Marks JR, Olson JA. Prognostic significance of the number oflymph nodes examined in patients with lymph node-negative breast carcinoma. Cancer. 2001; 91:2258-2262.

19. Tausch C, Taucher S, Dubsky P, Seifert M, Reitsamer R, Kwasny W, Jakesz R, Fitzal F, Filipcic L, Fridrik M, Greil $\mathrm{R}$, Gnant M. Prognostic value of number of removed lymph nodes, number of involved lymph nodes, and lymph node ratio in 7502 breast cancer patients enrolled onto trials of the Austrian Breast and Colorectal Cancer Study Group (ABCSG). Ann Surg Oncol. 2012; 19:1808-1817.

20. Camp RL, Rimm EB, Rimm DL. A high number of tumor free axillary lymph nodes from patients with lymph node negative breast carcinoma is associated with poor outcome. Cancer. 2000; 88:108-113.

21. van der Wal BC, Butzelaar RM, van der Meij S, Boermeester MA. Axillary lymph node ratio and total number of removed lymph nodes: predictors of survival in stage I and II breast cancer. Eur J Surg Oncol. 2002; 28:481-489.

22. Camp RL, Dolled-Filhart M, Rimm DL. X-tile: a new bioinformatics tool for biomarker assessment and outcomebased cut-point optimization. Clin Cancer Res. 2004; 10:7252-7259.

23. Zhuo C, Ying M, Lin R, Wu X, Guan S, Yang C. Negative lymph node count is a significant prognostic factor in patient with stage IV gastric cancer after palliative gastrectomy. Oncotarget. 2017 Apr 26. http://doi.org/10.18632/ oncotarget.17430. [Epub ahead of print].

24. Li Q, Zhuo C, Cai G, Li D, Liang L, Cai S. Increased number of negative lymph nodes is associated with improved cancer specific survival in pathological IIIB and IIIC rectal cancer treated with preoperative radiotherapy. 
Oncotarget. 2014; 5:12459-12471. http://doi.org/10.18632/ oncotarget. 2560 .

25. Wang H, Zhang C, Kong L, Zhu H, Yu J. Better survival in PMRT of female breast cancer patients with $>5$ negative lymph nodes: A population-based study. Medicine (Baltimore). 2017; 96:e5998.

26. Vrdoljak DV, Ramljak V, Muzina D, Sarceviç B, Knezević F, Juzbasić S. Analysis of metastatic involvement of interpectoral (Rotter's) lymph nodes related to tumor location, size, grade and hormone receptor status in breast cancer. Tumori. 2005; 91:177-181.

27. Chen M, Palleschi S, Khoynezhad A, Gecelter G, Marini CP, Simms HH. Role of primary breast cancer characteristics in predicting positive sentinel lymph node biopsy results: a multivariate analysis. Arch Surg. 2002; 137:606-9.
28. Cao Y, Paner GP, Rajan PB. Sentinel node status and tumor characteristics: a study of 234 invasive breast carcinomas. Arch Pathol Lab Med. 2005; 129:82-84.

29. Surveillance, Epidemiology, and End Results (SEER) Program (www.seer.cancer.gov) SEER*Stat Database: Incidence - SEER 18 Regs Research Data + Hurricane Katrina Impacted Louisiana Cases, Nov 2015 Sub (19732013 varying) - Linked To County Attributes - Total U.S., 1969-2014 Counties, National Cancer Institute, DCCPS, Surveillance Research Program, Surveillance Systems Branch, released April 2016, based on the November 2015 submission. 